\title{
Cavernous Haemangioma of the Adrenal Gland: A Case Report with Review of Literature
}

VENKATA SUBBAIH ARUNACHALAM'1 ${ }^{1}$ RAHUL DEV ${ }^{2}$, MOHIT TAYAL $^{3}$, SAKSHI GARG $^{4}$

\section{ABSTRACT}

Cavernous haemangioma of adrenal gland are rare benign, non-functioning tumours. They are incidental finding in the majority of cases with few cases detected at an earlier stage by virtue of having functional status. On imaging they have a characteristic temporal sequence of contrast enhancement from peripheral nodular to centripetal fill-in. Atypical enhancement patterns arise due to presence of large areas of necrosis within. The lesion might cause mass effect on adjacent structures on attaining large size, however shows no tendency to invade adjacent organs or vascular structures. The present case is of cavernous haemangioma in an elderly male patient presenting as vague right lumbar lump has been reported. CECT abdomen revealed a large necrotic right adrenal mass lesion suspicious for malignancy. The tumour was surgically removed with histopathological findings compatible with haemangioma. Even though rare; cavernous haemangiomas should always be kept in mind for differential of any adrenal mass. Low frequency of occurrence and lack of specific symptoms makes these tumours to be invariably diagnosed postoperatively.

Keywords: Adrenal lesion, Centripetal fill-in, Malignancy, Peripheral nodular enhancement

\section{CASE REPORT}

A 79-year-old male patient with complaints of non-progressive dull right flank pain for the last 2 months reported to the clinic. There were no associated constitutional symptoms with no loss of weight or appetite. The general physical examination and blood profile including hormone levels were within normal limits. A $2 \times 2 \mathrm{~cm}$ soft to firm mass was palpable in the right lumbar region. The patient underwent Contrast Enhanced Computed Tomography (CECT) after the written informed consent, ensuring renal parameters is within normal limits. The CECT showed a large round to oval heterogeneous mass lesion measuring approximately $7.6 \times 7.0 \times 7.3 \mathrm{~cm}$ in dimensions located in right suprarenal region with non-visualisation of the right adrenal gland. There were no appreciable areas of fat attenuation or haemorrhage within [Table/ Fig-1]. Post-contrast images demonstrated peripheral nodular enhancement on arterial phase progressing to partial centripetal fill-in on venous and delayed phases. Even on delayed phase scan, the entire lesion did not show contrast enhancement or fill in attributable to the large area of necrosis. There was compression, but no frank invasion of adjacent liver parenchyma. The right kidney was displaced inferiorly by the mass with maintained intervening planes [Table/Fig-2]. Contralateral kidney and adrenal gland were unremarkable. The IVC and right renal vein showed no filling defect. Surgical removal of the right suprarenal gland was done considering suspicion of malignant aetiology. The intra and postoperative period were uneventful. On gross examination, the mass was globular in shape with a smooth surface. The cut surface reveals haemorrhagic and cystic areas within. Microscopic examination revealed well encapsulated tumour showing dilated vascular spaces lined by endothelial cells along with areas of necrosis as well as presence of cortical cells in vicinity [Table/Fig-3]. The patient is stable and symptom free since 6 months and on follow up till date.

\section{DISCUSSION}

Cavernous haemangioma of the adrenal gland is an uncommon benign neoplasm commonly encountered in $5^{\text {th }}$ to $7^{\text {th }}$ decade with a female preponderance. The majority of lesions are unilateral and hormonally inactive and found incidentally. Histologically

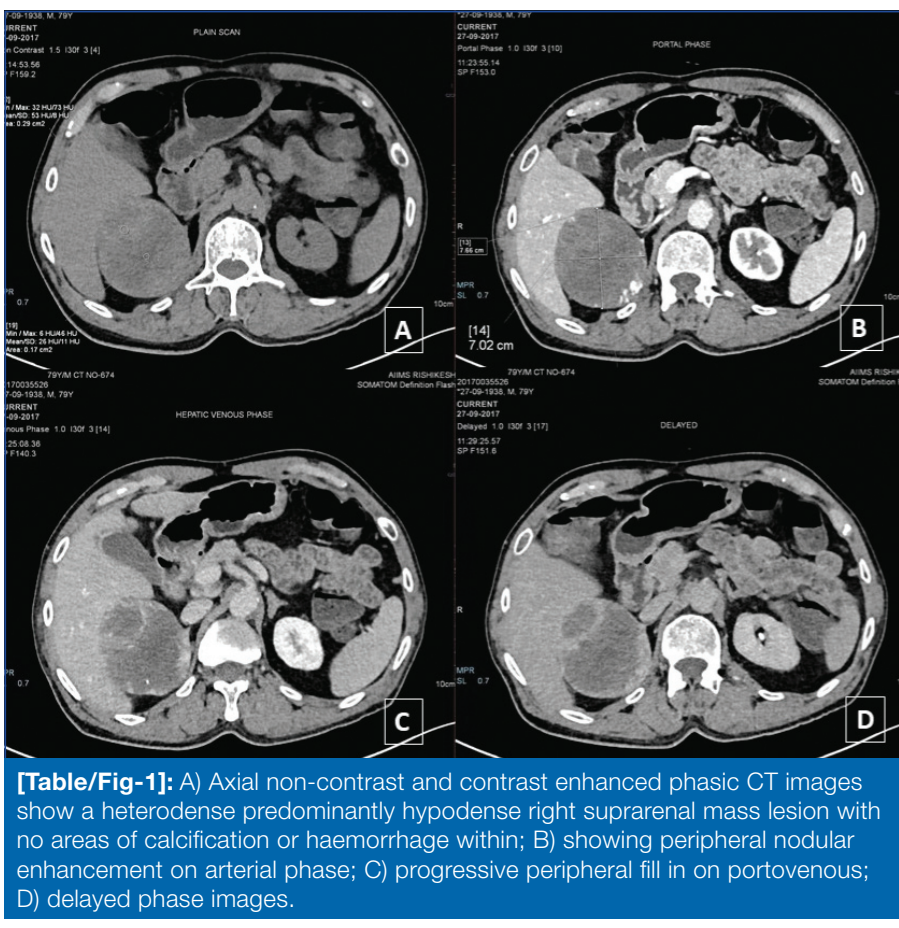

majority are of cavernous morphology composed of degenerating endothelium lining dilated vascular spaces. The first report was published in 1955 by Johnson CC and Jeppesen FB [1]. Since then approximately 62 further cases of the same have been encountered in the literature [2]. The associated clinical signs and symptoms are non-specific like heaviness or dragging sensation in lumbar region, which on examination may manifest as a palpable lump, similar features were also observed in this case [3]. Few cases of hormone secreting tumours are also mentioned in the past literature [4]. The present case had no signs and symptoms raising suspicion of hormonal dysfunction. Other manifestation may include coagulopathy. Rarely the lesion may present as painful mass or life threatening hypotension secondary to spontaneous or traumatic rupture [5]. Its incidence in the suprarenal gland has remained limited to only around 64 cases over the past six decades. Ultrasonography is the first line modality for any 

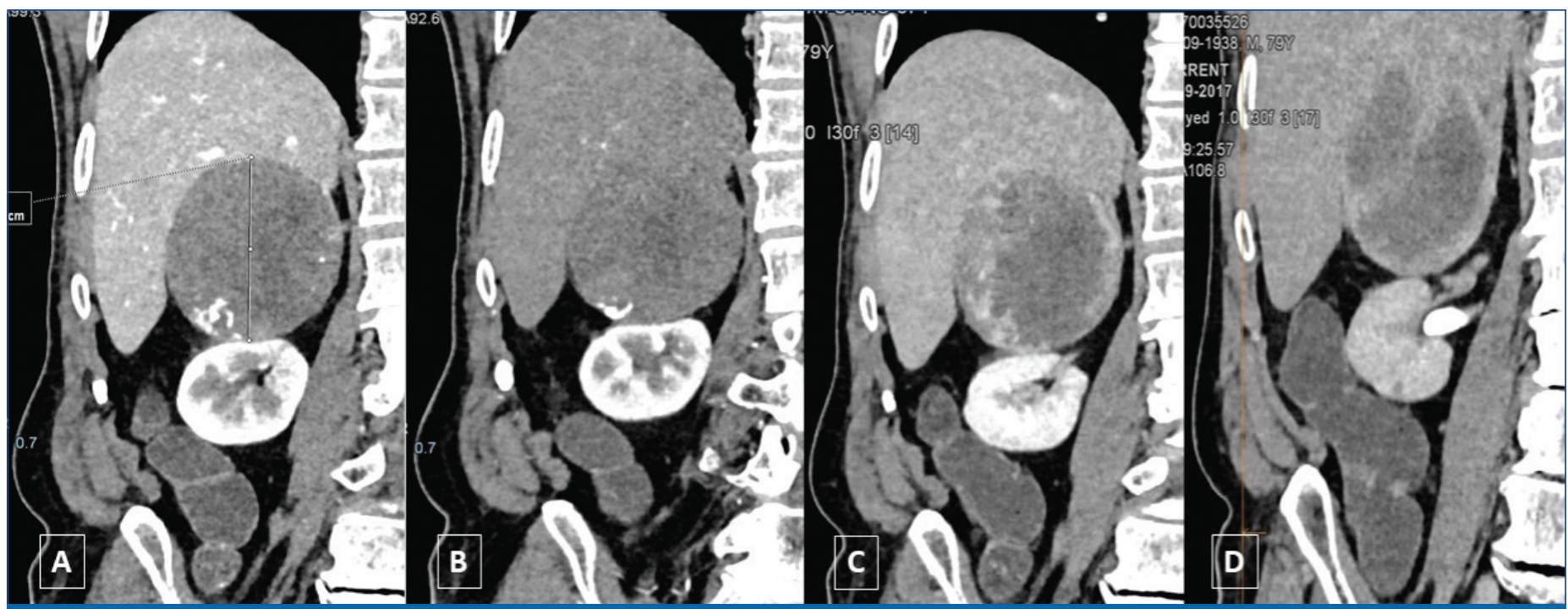

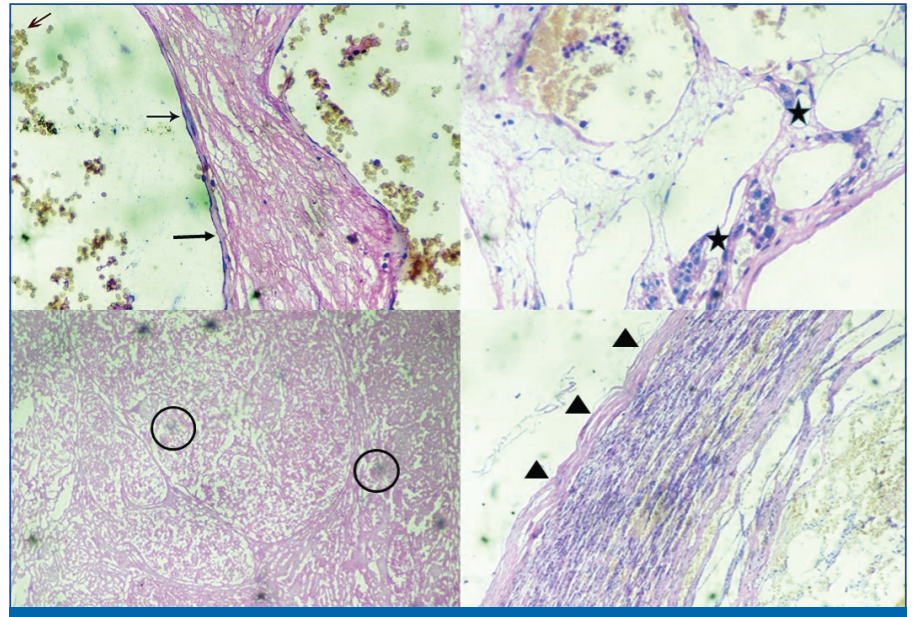

[Table/Fig-3]: Haematoxylin and eosin stained photomicrograph shows endothelial cells lining vascular spaces (black arrows), well differentiated zona reticularis cells adjacent to cavernous spaces (black asterisk), focal areas of necrosis (black circle) and limiting basement membrane (black arrowheads)

suspected suprarenal lesion and invariably done as a screening. Unfortunately, the imaging findings are nonspecific showing a heteroechoic lesion superior to kidney [6]. CT scans usually display a large heterogenous mass lesion with areas of hypodensity and foci of calcification showing either central or random peripheral distribution pattern. These foci of calcifications are pleomorphic in terms of both individual shape as well as distribution pattern within the lesion, representing phleboliths within dilated vascular spaces. The presence of calcification is a nonspecific finding seen in other lesions like haemorrhage, tuberculosis, cortical carcinoma and rarely metastasis. On postcontrast scan the lesions show heterogenous enhancement with early peripheral, central, or mixed patterns. The enhancement shows characteristic temporal progression from peripheral nodular in arterial phase to progressive centripetal fills in on portovenous and delayed phases [7]. Rare contrast enhancement patterns inciting dilemma include delayed washout pattern mimicking adrenal cortical carcinoma, peripheral thin rim of contrast enhancement as well as the complete absence of enhancement. The latter was seen in case encountered by Pang $C$ et al., due to entire lesion being necrotic [8]. The appearance on magnetic resonance imaging is in line with CECT representing different components of the lesion. Noncontrast sequences may show heterointense mass lesion in majority with areas of $\mathrm{T} 1$ hypointensity showing corresponding T2 hyperintensity representing necrosis. There can also be focal areas of $\mathrm{T} 1$ hyperintensity representing haemorrhage as well as T1 and T2 hypointense areas representing calcifications [3,9,10]. Although the above mentioned findings are non-specific, increase the diagnostic confidence when combined with clinical context and CECT findings. Above mentioned findings can also be seen in other adrenal neoplasms and are not exclusive to adrenal haemangioma. Angiography if done may show peripheral pooling of contrast persisting into the venous phase $[11,12]$. With the typical pattern of peripheral nodular enhancement progressing to progressive centripetal fill the diagnosis of haemangioma is almost certain. Presence of calcifications in a more diffuse distribution further confirms the diagnosis. The large size of the lesion with areas of necrosis also raises sinister diagnosis like cortical carcinoma and pheochromocytoma. Other differentials include haematoma, ganglioneuroma and metastasis [13,14]. The main factor while contemplating surgery is the size of the tumour for two reasons, first increased risk of spontaneous rupture leading to life threatening haemorrhage and second risk of harbouring an underlying malignancy. Other factors to be considered include symptoms of mass effect, functional lesions, interval growth on comparative imaging and atypical contrast enhancement characteristics. Most of the tumours are usually removed via open surgery with laparoscopic approach used in a few cases [11]. The validity of a laparoscopic approach in cases suspicious for malignancy is debatable. Grossly, the tumour is usually firm, well circumscribed and encapsulated. The cut section of the mass shows heterogenous internal contents with large areas of haemorrhage or clotted blood. These lesions are based on the adrenal cortex, composed of dilated blood filled sinusoidal spaces lined by single layer of endothelial cells [15]. They are classified into cavernous and capillary types with former having larger and disorganised peripherally placed blood filled spaces and latter having clusters of radially arranged capillaries. On immunohistological examination, the tumour invariably stains positive for CD31, CD34 and factor VIII conforming endothelial origin. They are thought to be congenital lesions having natural course of enlargement over time. Pitfalls in histopathological evaluation include inadequate assessment of subcapsular area and loss of architecture from extensive necrosis or haemorrhage leading to erroneous diagnosis of carcinoma and cyst respectively.

\section{CONCLUSION}

Cavernous haemangioma arising from right adrenal gland is a rare benign tumour, and it should be considered in differential of adrenal mass lesions, especially those which are heterogenous and show areas of calcification within. The typical pattern of contrast enhancement can suggest, but not definitively diagnose it. This case was unique as it showed atypical pattern of contrast enhancement on CECT with large area of central necrosis and no evidence of rupture in spite of very large size. The definite treatment is surgical 
removal followed by histopathological confirmation especially in larger lesions prone for rupture as well as for the definite exclusion of underlying malignancy. The current case was successfully treated by open resection without any complications and uneventful follow-up.

\section{REFERENCES}

[1] Johnson CC, Jeppesen FB. Hemangioma of the adrenal. J Urol. 1955;74:57377.

[2] Kieger AJ, Nikolaidis P, Casalino DD. Adrenal gland hemangioma. Journal of Urology. 2011;186 (December (6):2415-16 [Epub 2011 Oct 21].

[3] Harzallah L, Zouari L, Ben Cherifa L, Harzallah F, Sriha B, Bakir D, et al. Hémangiome surrénalien: à propos d'un cas. Annal Endocrinol. 2006;67:624-27.

[4] Oishi M, Ueda S, Honjo S, Koshiyama H, Yuba Y, Takabayashi A. Adrenal cavernous hemangioma with subclinical Cushing's syndrome: Report of a case. Surgery Today. 2012;42:973-77.

[5] Forbes TL. Retroperitoneal haemorrhage secondary to ruptured cavernous hemangioma. Can J Surg. 2005,48:78-79.

[6] Boraschi P, Campatelli A, Di Vito A, Perri G. Hemorrhage in cavernous haemangioma of the adrenal gland: US, CT and MRI appearances with pathologic correlation. European Journal of Radiology. 1995;21:41-43.
[7] Yamada T, Ishibashi T, Saito H, Majima K, Tsuda M, Takahashi S, et al. Two cases of adrenal hemangioma: CT and MRI findings with pathological correlations. Radiat Med. 2002;20:51-56.

[8] Pang C, Wu P, Zhu G. A rare cavernous hemangioma of the adrenal gland. Urology Case Reports. 2015;3(4):120-22.

[9] Hamrick-Turner JE, Cranston PE, Shipkey FH. Cavernous hemangioma of the adrenal gland: MR findings. Magnetic Resonance Imaging. 1994;12:1263-67.

[10] Silverberg D, Paramesh AS, Roayaie S, Schwartz ME. Giant hemangioma of the adrenal gland. Israel Medical Association Journal. 2004;6:705-06.

[11] Thiele JW, Bodie B. Adrenal hemangioma. Surgery. 2001;129:373-74.

[12] Rothberg M, Bastidas J, Mattey WE, Bernas E: Adrenal haemangiomas: angiographic appearance of a rare tumor. Radiology. 1978;126:341-44.

[13] Terzolo M, Stigliano A, Chiodini I, Loli P, Furlani L, Arnaldi G, et al. AME position statement on adrenal incidentaloma. European Journal of Endocrinology/ European Federation of Endocrine Societies. 2011;164:851-70.

[14] Johnson PT, Horton KM, Fishman EK. Adrenal mass imaging with multidetector CT: Pathologic conditions, pearls, and pitfalls. Radiographics. 2009;29:1333-51.

[15] Telem DA, Nguyen SQ, Chin EH, Weber K, Divino CM. Laparoscopic resection of giant adrenal cavernous hemangioma. JSLS: Journal of the Society of Laparoendoscopic Surgeons/Society of Laparoendoscopic Surgeons. 2009;13:260-62.

\section{PARTICULARS OF CONTRIBUTORS:}

1. Junior Resident, Department of Radiodiagnosis and Imaging, All India Institute of Medical Sciences, Rishikesh, Uttarakhand, India.

2. Assistant Professor, Department of Radiodiagnosis and Imaging, All India Institute of Medical Sciences, Rishikesh, Uttarakhand, India.

3. Senior Resident, Department of Radiodiagnosis and Imaging, All India Institute of Medical Sciences, Rishikesh, Uttarakhand, India.

4. Senior Resident, Department of Pathology, All India Institute of Medical Sciences, Rishikesh, Uttarakhand, India.

\section{NAME, ADDRESS, E-MAIL ID OF THE CORRESPONDING AUTHOR}

Dr. Rahul Dev,

Flat No-5/1, Building No-57, AllMS, Rishikesh Residential Complex-249203, Rishikesh, Uttarakhand, India.

E-mail: rdev8283@gmail.com 